\title{
ANALYTICAL SOLUTIONS FOR SQUARE SHAPE PRESSURE MICROSENSORS
}

\author{
Sumit Kumar Jindal ${ }^{1}$, Sanjeev Kumar Raghuwanshi ${ }^{2}$ \\ ${ }^{I}$ Department of Electronics Engineering, Indian School of Mines, Dhanbad, 826004, India \\ ${ }^{2}$ Department of Electronics Engineering, Indian School of Mines, Dhanbad, 826004, India
}

\begin{abstract}
Most commonly for MEMS Systems the shapes used for diaphragm are square, circular or rectangular. Here square shape is under consideration. Pressure microsensor designed is operated in linear range. All the analytical solutions are presented along with set of simple equations to calculate and predict the sensitivity of pressure micro sensors. By the use of COMSOL and simulation it can be found that analytical solutions hold good.
\end{abstract}

Keywords: Microsensors, sensitivity, Burst pressure, piezoresisive

\section{INTRODUCTION}

Miniaturisation and integration of electronic devices have made far reaching technological revolutions. Integration of micromechanics on the same chip has resulted in MicroElectro-Mechanical System(MEMS) that have added newer dimensions to conventional ICs. With the advent of micromachining technology, which is compatible with the conventional IC industry, MEMS has become dominant in many areas of applications. MEMS have main applications in sensors, actuators and smart structures. [1]

One of the most common MEMS device is Pressure microsensors. Due to demand for lower cost and smaller dimensions transition from mechanical to silicon type has occurred.[2] Sensor sensitivity and burst pressure determines the diaphragm dimensions. By burst pressure we mean maximum non destructive pressure. In a piezoresistive device, when a silicon microdiaphragm is subjected to uniform pressure, its internal strain changes and deflection occurs. Silicon being a piezoresistive material due to change in internal strain its resistance changes.. Now pressure can be measured by continuous check of resistance change by the help of pressure sensing resistors. This change is then converted to its equivalent electrical output signal. Thin plate problem can be used to simulate the diaphragm.[3]

In this study, the stress is related to diaphragm size, burst pressure, pressure sensitivity and piezoresistor length and these corresponding factors are the main design parameters. The present work offer simple and valid expressions for designers and researchers to predict the piezoresistive microsensors.

\section{NUMERICAL ANALYSIS}

A resistor bridge is all that a piezoresistive sensor having square shape contains. The basic assumptions for kirchoff's plate theory are[4]

a) The plate material is elastic, isotropic and homogenous, b) Plate is flat in nature,

c) Deflection is very small compared to the thickness of the plate; therefore slope is small and hence square of slope is negligible.

d) the strain line remain straight and normal to the middle surface during the deformation

e) In the stress strain relation stress normal to the middle plane is small compared with the other stress components and for this reason it is neglected

f) Plate displacement is small and it is also assumed that after bending middle surface remains unstrained

The deflection of the flat diaphragm under uniform pressure load can be found by solving the differential equation

$$
\frac{\partial^{4} w}{\partial x^{4}}+2 \frac{\partial^{4} w}{\partial x^{2} \partial y^{2}}+\frac{\partial^{4} w}{\partial y^{4}}=\frac{P}{D}
$$

Where $\mathrm{D}$ is the flexural rigidity given by $\frac{E h^{3}}{12\left(1-v^{2}\right)}$. By solving (1) with boundary conditions we get:

$$
w=0, \frac{\partial w}{\partial n}=0
$$

\section{RESULTS AND DISCUSSIONS}

\subsection{Diaphragm Size}

The maximum deflection is found to be at the centre of the diaphragm.[5-7] 

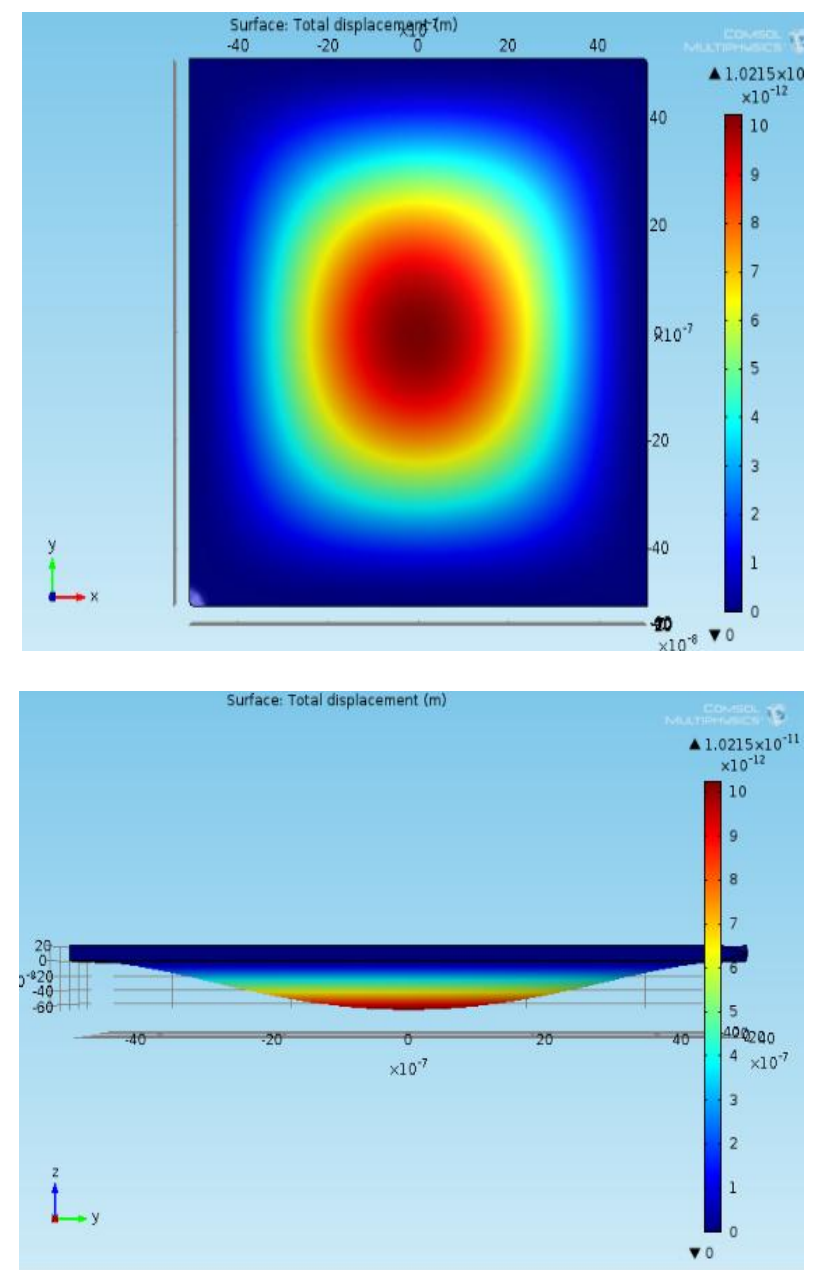

Fig-1: Lateral and front plane view of displacement for the square diaphragm in agreement to the theory

The maximum deflection of diaphragm is linear to pressure applied. The diaphragm thickness is about some ten micrometres and the deflection is less than half of the diaphragm thickness.

\subsection{Burst Pressure}

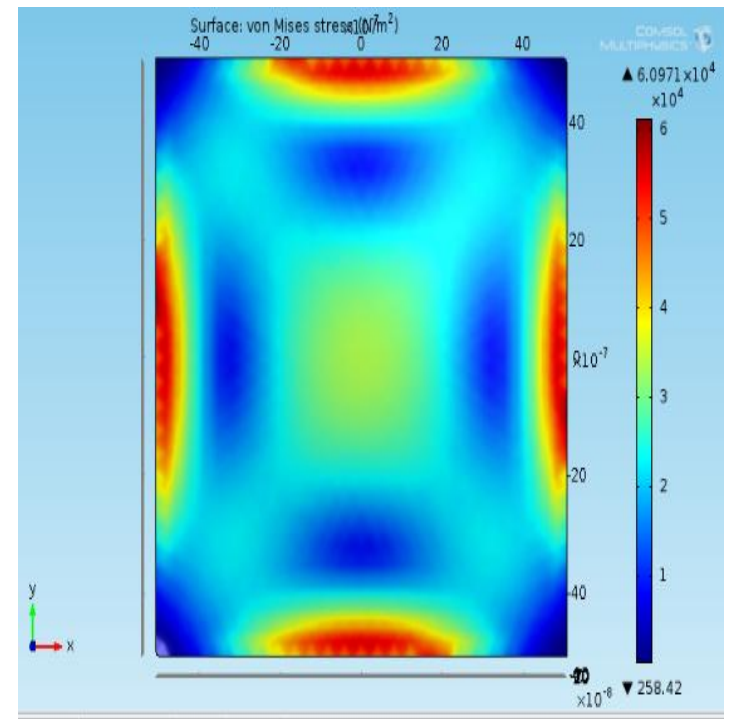

Fig-2: Lateral view of stress for the square diaphragm in agreement to the theory stated
At the middle of the edges the maximum stress occurs

\subsection{Pressure Sensitivity}

Parallel and perpendicular to the direction of the electrical current, piezoresistivity of silicon can be studied. Gauge factor can be represented as Fpar and Fper. Piezoresistive constant $\pi$ is used to represent them as[1],[8]

$$
\begin{gathered}
F_{p a r}=\frac{E\left(\prod_{l}+v \prod_{t}\right)}{\left(1-v^{2}\right)} \\
F_{p e r}=\frac{E\left(\prod_{t}+v \prod_{l}\right)}{\left(1-v^{2}\right)}
\end{gathered}
$$

And the change in resistance is written as:

$$
\frac{\Delta R}{R}=F_{p a r} \varepsilon_{r}+F_{p e r} \varepsilon_{t}
$$

Which can further be written a

$$
\frac{\Delta R}{R}=\left(F_{p a r}+\frac{1}{1-v}\right) \varepsilon_{r}+\left(F_{p e r}+\frac{1-2 v}{1-v}\right) \varepsilon_{t}
$$

\subsection{Optimization of Resistor Length}

Important positions for sensing resistors are analyzed after the calculation of sensitivity distribution. Then we calculate the design of the resistors which includes shape and length for the effective calculation of optimized sensitivity. Line shape resistors are designed for change in strain to calculate the maximum piezoresistive effect. In order to gain maximum sensitivity the line shape resistors are placed at the edges of the square diaphragm. The non-effective portion used is $2 \mu \mathrm{m}$ outside the diaphragm. This is taken into consideration because misalignment cannot be avoided during the process of fabrication. During pressure measurement the sensor parts which are effective will stay inside diaphragm. 


\subsubsection{Configuration of the Piezoresistive Pressure}

\section{Sensor}

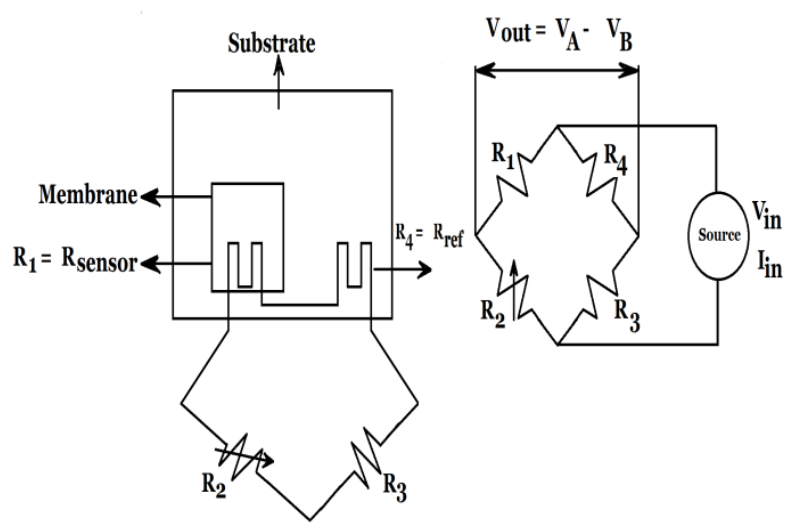

(a)

(b)

Figure-3:a) The physical configuration of the pressure sensor and circuit fabricated on a membrane suspended on a

$\mathrm{Si}$ substrate. Rsensor is a piezoresistor that changes resistance with the strain of the membrane, across which a pressure differential is applied. b):The wheatstone quarter bridge circuit used to sense the change in resistance of Rsensor.

Figure (3a) and (3b) shows the circuit design for the square shaped diaphragm pressure sensor. It also shows the wheatstone bridge circuit used for signal conditioning of pressure sensor. Here a quarter sensor bridge is employed. It is enough to demonstrate the strain effect on the sensor. $R_{1}$ is the piezoresistor on which membrane is embedded and pressure difference is applied. To nullify the output voltage $V_{\text {out }}=V_{A}-V_{B}$, a variable resistor $R_{2}$ is used. This is the case when no pressure is applied across the membrane. For balancing of bridge $R_{3}$ is used. $R_{4}$ is the reference resistor. Since wheatstone bridge has maximum sensitivity for the output voltage when variable resistance is used hence it is used in this configuration. A constant voltage (RMS or dc), $V_{i n}$, is used as input voltage source.

\subsubsection{Sensor Output Analysis (When a Voltage is}

\section{Applied)}

For quarter bridge circuit the output is written as below provided no pressure is [9]

$$
V_{\text {out }}=\frac{R_{1} R_{3}-R_{2} R_{4}}{\left(R_{1}+R_{2}\right)\left(R_{3}+R_{4}\right)} V_{\text {in }}
$$

If all resistance is assumed to be $\mathrm{R}$ initially and then after we apply pressure Vout is written as:

$$
\frac{\Delta R}{2(2 R+\Delta R)} V_{i n}
$$

Where $\Delta \mathrm{R}$ is the change in resistance of $\mathrm{R}$.

So the output voltage can be expressed as

$$
V_{\text {out }}=\frac{\Delta R_{\text {eff }}}{2\left\{2\left(R_{\text {non }}+R_{\text {eff }}\right)+\Delta R_{\text {eff }}\right\}} V_{\text {in }}
$$

Where $\Delta R_{\text {eff }}$ is the change effective resistance. In order to get maximum voltage it is important to maximize $\Delta R_{\text {eff }}$ and $R_{n o n}$ is the value assigned to non-effective resistance. $R_{\text {eff }}$ is effective resistance.

It can be derived as:

$$
\frac{\Delta R_{e f f}}{R_{e f f}}=\frac{1}{l} \int_{L}^{L-l} \frac{\Delta R}{R} d x
$$

Where $l$ effective resistor's length

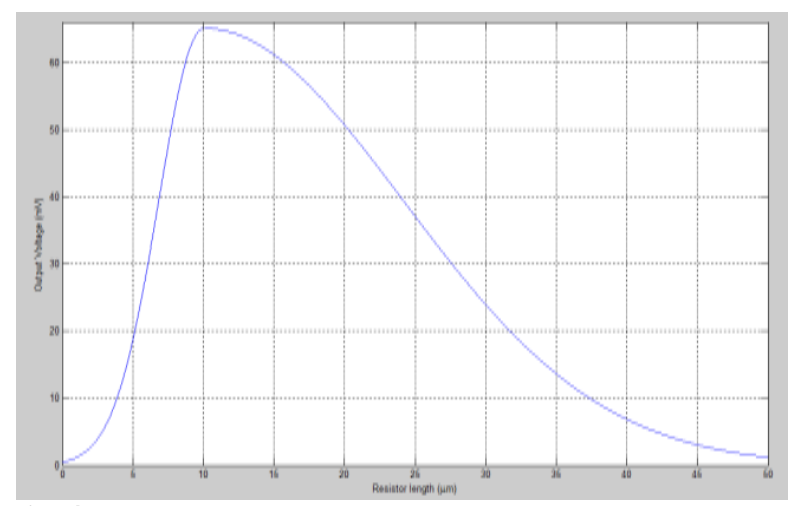

Fig-4: Optimization result of effective resistor length for square shaped diaphragm under consideration

Effective resistor's optimized length is calculated by the help eqn (7) \& (9). Fig.(4) shows the simulation result for the circular diaphragm with above mentioned dimensions. It is observed that diaphragm has an optimized output when the effective resistor length is about $10 \mu \mathrm{m}$. It decreases whether we increase or decrease the length.

\section{CONCLUSION}

In this paper we have provided all the analytical steps for the calculation of the freely supported case for square shaped pressure sensor. The results have been matched with the existing literature. Design and modelling of pressure sensor have been successfully established. We derived the relation among maximum diaphragm deflection, diaphragm configuration and applied pressure. The sensitivity has been expressed in terms of piezoresistive constants. The effect of piezoresistor has also been included in the model for more precise calculation. The orientation, length and position of the sensing resistors has completely been studied. For square shape diaphragms, at the centre lies the most sensing 
portions i.e at $10 \mu \mathrm{m}$. When the shape or dimension or orientation is changed for the diaphragm or resistor these solutions provide researchers with important design outlines. Moreover the literature has also been supported with simulation result of COMSOL.

\section{REFERENCES}

[1]. Liwei Lin, Weijie Yun,1998.MEMS pressure sensors for Aerospace Applications.IEEE 0-7803-4311-5/98, 429436

[2]. Smith,C.S., 1954.Piezoresistive Effect in Germanium and Silicon. phys. Rev. 94, 42-49

[3]. Timoshenko,S.P., Woinowsky-Kreiger,S.,1959. Theory of Plates and Shells, Second ed. McGraw Hill, New York

[4]. Carl T.F Ross, 1999. Strength of Mateial and Structure, fourth ed. Arnold,London.

[5]. William P. Eaton 'et al'.,1999. A new analytical solution for Diaphragm Deflection and its application to a surface- micromachined Pressure Sensor. International Conference on Modelling and Simulation of Microsystems [6]. DING Hao-jiang 'et al'.,2005. Analytical solutions for a uniformly loaded circular plate with clamped edges. Journal of Zhejiang university Science A(10), 1163-1168

[7]. Khakpour,R.,'et al'.,2010. Analytical comparison for square, Rectangular and Circular Diaphragms in MEMS applications. International Conference on Electronic Devices. Systems and Applications, 297-99.

[8]. Lin L 'et al'.,1999. A simulation program for the sensitivity and linearity of piezoresistive pressure sensors. J. Micro-electromech. Syst. 8, 514-22.

[9]. Byunghoon Bae 'et al'.,2004. Design optimization of a piezoresistive pressure sensor considering the outputsignalto-noise ratio. J. Micromech. Microeng. 14, 1597-1607 